\title{
Neuromodulación de raíces sacras como tratamiento de la incontinencia fecal. Resultados preliminares
}

\author{
J. M. Navarro, A. Arroyo Sebastián, F. Pérez Vicente, A. M. Sánchez Romero, J. Pérez Legaz, P. Serrano Paz, \\ A. M. Fernández Frías, F. Candela Polo y R. Calpena Rico \\ Unidad Coloproctología. Servicio de Cirugía General y del Aparato Digestivo. Hospital General Universitario. Elche, \\ Alicante
}

\section{RESUMEN}

Presentamos nuestra experiencia inicial en el tratamiento de la incontinencia fecal (IF) mediante neuromodulación de raíces sacras (NRS), a través de los resultados de un estudio prospectivo realizado con 26 pacientes en el que se comparan los valores basales en la escala de continencia de Wexner-Cleveland y en la capacidad para el retraso de la defecación, con los obtenidos tras un año de terapia con NRS. El estudio inicial de cada paciente incluía anamnesis, exploración general, ecografía y manometría rectal, así como unos diarios de continencia y de calidad de vida específicos para la IF de 3 semanas. Antes de la terapia con NRS, el valor medio en la escala Wexner-Cleveland fue de 15,00 $\pm 1,81$ y el $62,50 \%$ de los pacientes tenía una capacidad de retraso de la defecación menor de 1 minuto. Tras un año de terapia con NRS, el valor medio en la escala de Wexner-Cleveland fue de 4,87 $\pm 2,54$ ( $p=0,0031$ ) y el $75,01 \%$ de los pacientes presentaba una capacidad de retraso defecatorio mayor de 15 minutos ( $p=0,0018$ ). Hacemos, además, una descripción detalla de la técnica quirúrgica de la NRS, haciendo referencia a sus indicaciones y finalizamos revisando las distintas opciones terapéuticas para la IF mostrando nuestro algoritmo terapéutico para esta patología. La NRS es una técnica eficaz para el tratamiento de la IF en pacientes seleccionados que no han respondido a tratamiento conservador, biofeedback o correcciones anatómicas (esfinteroplastia), con una mínima morbilidad y susceptible de realizarse en un programa de cirugía ambulatoria.

Palabras clave: Incontinencia fecal. Neuromodulación. Raíces sacras.

\begin{abstract}
We present our initial experience in the treatment of fecal incontinence (FI) with sacral root neuromodulation (SRN) by reporting the results of a prospective study with 26 patients where baseline Wexner-Cleveland scale scores and ability to delay defecation were compard to results after one year with SRN. The initial study of patients included history taking, general examination, anal ultrasonography, and manometry, and a three-week diary of continence and quality of life specific for FI was used. Before SRN the mean baseline Wexner-Cleveland score was $15.00 \pm 1.81$, and $62.50 \%$ of patients could only delay defecation for less than a minute. After a year with NRS the mean Wexner-Cleveland score was $4.87 \pm 2.54$ ( $p=0.0031)$, and $75.01 \%$ of patients could delay defecation above fifteen minutes $(p=0.0018)$. We also describe the surgical technique and its indications, and finally review the various therapeutical options for FI and show our algorithm for this condition. SRN is an effective technique for the treatment of FI in properly selected patients with no response to medical therapies (including biofeedback) or anatomic correction (sphincteroplasty), with efficacy, little morbidity, and a short hospital stay.
\end{abstract}

Key words: Fecal incontinente. Neuromodulation. Sacral roots.

Navarro JM, Arroyo Sebastián A, Pérez Vicente F, Sánchez Romero AM, Pérez Legaz J, Serrano Paz P, Fernández Frías AM, Candela Polo F, Calpena Rico R. Neuromodulación de raíces sacras como tratamiento de la incontinencia fecal. Resultados preliminares. Rev Esp Enferm Dig 2007; 99: 636-642.

\section{INTRODUCCIÓN}

La incontinencia fecal (IF) se define como la incapacidad de diferir la defecación hasta que se encuentra el lu-

Recibido: 20-05-07.

Aceptado: 10-09-07.

Correspondencia: José Manuel Navarro Rodríguez. C/ Limoneros, 48. 03160 Almoradí, Alicante.e-mail: jomanarrocir@hotmail.com gar y el momento socialmente idóneos para realizarla, produciéndose una pérdida recurrente e involuntaria de material fecal o gases a través del canal anal. Aún en los casos más leves de IF (en donde únicamente se producen escapes y ensuciamientos de la ropa interior), esta patología conlleva en mayor o menor medida problemas físicos, psicológicos y alteraciones en la calidad de vida de los pacientes (en los ámbitos sexual, familiar, social y laboral) (1-3). 
El hecho de que la IF haya sido considerada socio-culturalmente como un problema vergonzante, dificulta la estimación de su prevalencia real, la cual aumenta con la edad y afecta predominantemente a mujeres (3). Estudios epidemiológicos españoles estiman una prevalencias del $13,6 \%$ en mayores de 65 años y del $8 \%$ en mujeres mayores de 18 años (4).

El aumento de la demanda asistencial de la IF debido tanto al envejecimiento en las pirámides poblacionales de los países desarrollados, como al mayor acceso a la información médica, ha condicionado un resurgimiento en el interés médico en esta patología, en el que los recursos terapéuticos históricamente han sido limitados y no demasiado efectivos (4). La esfinterotomía hasta hace poco era la única opción quirúrgica indicada exclusivamente para pacientes con lesiones esfintéricas y cuya eficacia terapéutica oscila entre el 50-60\% a largo plazo según las series (5); por contrapartida, el vacío y nihilismo terapéutico para los pacientes sin defectos estructurales esfintéricos, les relegaba a la opción de la colostomía para los casos más graves (6). En los últimos años, como resultado del desarrollo en el campo de la neurofisiología defecatoria, han aparecido nuevas opciones terapéuticas para la IF, las cuales han logrado un mayor éxito de resultados clínicos en esta patología (6). Así, la neuromodulación de raíces sacras (NRS), con eficacia clínica demostrada ampliamente en la incontinencia urinaria $(7,8)$, surge como una opción terapéutica reciente y en desarrollo para el tratamiento de la IF (9). A pesar de los escasos estudios publicados (no más de 300 casos en la bibliografía internacional), los resultados iniciales nos sugieren que nos encontramos ante una terapia eficaz con un prometedor futuro, y que las series extensas con seguimientos a largo plazo, acabarán por situarla en un lugar predominante dentro del arsenal terapéutico de la IF (9-16). Presentamos nuestra experiencia inicial en el tratamiento de la IF mediante la NRS a través de los resultados de un estudio prospectivo realizado con 26 pacientes en cuanto a la variación en la escala de continencia de Wexner-Cleveland y a la capacidad para el retraso de la defecación tras un año de terapia con NRS, realizando además una revisión de las distintas opciones terapéuticas para la IF y mostrando nuestro algoritmo terapéutico para esta patología.

\section{MATERIAL Y MÉTODO}

Estudio prospectivo transversal de 26 pacientes tratados en nuestro centro con NRS durante el periodo comprendido entre marzo de 2002 y enero de 2007. Fueron seleccionados para el tratamiento con NRS aquellos pacientes con esfínter anal externo indemne o reparado, y que a pesar de medidas higiénico-dietético-farmacológicas, persistían con criterios de IF para sólidos o líquidos de al menos un episodio de incontinencia semanal. Los criterios de exclusión para el estudio fueron: embarazo, menores de 18 años, infección aguda-crónica local, coa- gulopatías, marcapasos o arritmias cardiacas, lesión medular o neuropatías sistémicas, enfermedad oncológica, portadores de colostomía y pacientes con alteraciones psiquiátricas que imposibilitaran la competencia para responder adecuadamente al protocolo. El estudio basal de los pacientes subsidiarios de NRS comprendía: historia clínica general; diario de continencia de 3 semanas con los parámetros necesarios para obtener la puntuación en la escala de continencia de Wexner-Cleveland (Tabla I) e información acerca del hábito y la consistencia deposicional; dos cuestionarios de calidad de vida (el genérico SF-36 y el específico para la IF de la Sociedad Americana de Cirugía Colorrectal, ASCRS) $(17,18)$ así como manometría anal y ecografía endoanal $(16,17)$. Por otro lado, también se valoró la capacidad para el retraso defecatorio distribuyendo la aparición de la IF en 5 periodos: menos de 1 minuto, entre 1 y 5 minutos, entre 5 y 10 minutos, entre 10 y 15 minutos y finalmente, en más de 15 minutos.

Tabla I. Escala de Incontinencia Fecal de Wexner-Cleveland

\begin{tabular}{lccccc}
\hline & No & $\begin{array}{c}\text { Ocasional } \\
(<1 / \text { mes })\end{array}$ & $\begin{array}{c}\text { Semanal } \\
(<1 / \text { Fres, } \\
>1 / \text { sem })\end{array}$ & (> 1/sem) & \\
\hline Aire & 0 & 1 & 2 & 3 & 4 \\
Soiling & 0 & 1 & 2 & 3 & 4 \\
Heces sólidas & 0 & 1 & 2 & 3 & 4 \\
Compresa & 0 & 1 & 2 & 3 & 4 \\
Alteración estilo de vida & 0 & 1 & 2 & 3 & 4 \\
\hline
\end{tabular}

\section{Técnica quirúrgica de implantación de NRS: la terapia de NRS se divide en 3 fases}

1. Etapa de estimulación aguda. En esta etapa, tras estimular las raíces sacras S3 y S4 de ambos lados, se coloca el electrodo de estimulación en el foramen sacro de aquella raíz en la que se ha obtenido la mejor respuesta sensitiva-motora. Para su realización, se coloca al paciente en decúbito prono y en posición de navaja con el ano y los pies descubiertos. En condiciones de esterilidad y tras marcar con un rotulador cutáneo las referencias óseas para ubicar los forámenes sacros $\mathrm{S} 3$ y S4, se procede a la infiltración con anestésico local (mepivacaína al 2\%) del trayecto que recorrerá la aguja de estimulación eléctrica. A continuación, bajo control radioscópico, se introduce la aguja portadora del electrodo de estimulación de la raíz sacra, hasta ubicarla en el interior del foramen sacro. Posteriormente, se procede a la estimulación percutánea de cada una de las raíces sacras con una corriente eléctrica de $20 \mathrm{~Hz}$ y un voltaje creciente (de 1 a $10 \mathrm{~V}$ ). A medida que esto sucede, el cirujano preguntará al paciente si siente la estimulación eléctrica y además, observará si esta produce contracción en el ano, periné o dedos del pie. En la tabla II se resumen las respuestas sensitivo-motoras definidas para las raíces sacras S3 y S4. Así, de entre las 4 raíces, se selecciona en la que se obtiene una mejor respuesta sensitivo-motora con un menor voltaje de 
Tabla II.

\begin{tabular}{lccc}
\hline Raíz sacra & \multicolumn{2}{c}{ Respuesta motora } & Respuesta sensitiva \\
S3 3 Pélvico & $\begin{array}{c}\text { Contracción en "fuelle" } \\
\text { del periné (elevación } \\
\text { del suelo pélvico) }\end{array}$ & $\begin{array}{c}\text { Flexión plantar del } \\
\text { primer dedo del pie } \\
\text { (a veces del resto } \\
\text { de los dedos) }\end{array}$ & $\begin{array}{c}\text { Opresión en ano, } \\
\text { irradiada hacia genitales }\end{array}$ \\
S4 & $\Phi$ & $\begin{array}{c}\text { Opresión en ano } \\
\text { (exclusivamente) }\end{array}$ \\
\hline
\end{tabular}

estimulación. En dicha raíz, quedará colocado el electrodo de estimulación en el interior del foramen sacro correspondiente. A continuación, se realiza una incisión de $4 \mathrm{~cm}$ en la región glútea homolateral a la raíz estimulada, disecándose el tejido celular subcutáneo hasta alcanzar la fascia presacra y acceder al electrodo de estimulación. Dicho electrodo es conectado con un cable (cuyo remanente quedará alojado en un bolsón subcutáneo bajo la incisión glútea), el cual irá acoplado a un dispositivo portátil generador de corriente. A continuación, se programa el dispositivo generador de corriente externa y el paciente entra en la segunda etapa de la NRS.

2. Etapa de estimulación subcrónica o de cribado. En esta etapa, el paciente evalúa la efectividad de la corriente de estimulación generada por el dispositivo portátil, sobre el electrodo ubicado en el foramen sacro de la raíz sacra seleccionada en la etapa previa. Durante esta fase, de 3 semanas de duración, el paciente rellenará un nuevo diario de continencia que será comparado con el diario basal.

3. Etapa de estimulación crónica-definitiva. Únicamente aquellos pacientes en los que se haya logrado una reducción mínima del $50 \%$ en los episodios de IF durante la etapa de cribado, serán candidatos para la colocación del dispositivo de estimulación definitiva. Durante una breve intervención quirúrgica bajo anestesia local, se procederá a la reapertura de la incisión glútea para colocar una batería interna, la cual sustituirá al dispositivo externo y será la encargada de generar un estimulo eléctrico continuo sobre la raíz sacra.

\section{Seguimiento de los pacientes}

Los pacientes fueron revisados al mes, a los 6 meses y al año del implante del generador definitivo, evaluándose: los diarios de continencia y cuestionarios de calidad de vida rellenados nuevamente por los pacientes durante las 3 semanas previas a cada consulta; los parámetros de la corriente de estimulación (voltaje, frecuencia y amplitud, así como reprogramación de la misma si fuera necesario); y una manometría (realizada únicamente al primer mes del implante definitivo de la NRS). Se calculó el valor medio muestral al año de la NRS para la escala de continencia de Wexner-Cleveland y la capacidad para el retraso de la evacuación. Dichos parámetros fueron comparados con los valores previos a la terapia.

\section{Estudio estadístico}

Se emplearon las pruebas no paramétricas de rangos y signos de Wilcoxon y Mann-Whitney, para la realización del estudio estadístico comparativo de la continencia tras la NRS con respecto a los valores basales y al año de seguimiento en la escala Wexner-Cleveland, con un nivel de significación del 0,05. Para la comparación estadística de la continencia con respecto a la capacidad para retrasar la evacuación, se empleó una tabla de contingencia sobre la que se calculó el coeficiente Chi-cuadrado de Pearson, estableciendo igualmente un nivel de significación del 0,05.

\section{RESULTADOS}

Los datos del estudio irán expresados en media \pm desviación estándar salvo que se indique lo contrario. El implante definitivo de NRS fue colocado en 24 de los 26 pacientes incluidos en el estudio. Se desestimó la colocación en 2 pacientes debido a que no se observó mejoría clínica evidente (superior al 50\%) tras la aplicación percutánea temporal. Por ello, en el estudio estadístico para la comparación de variables tras la NRS (puntuación en la escala Wexner-Cleveland y capacidad de retraso de la defecación), incluimos exclusivamente a los 24 pacientes con implante definitivo de NRS.

En la tabla III mostramos de manera detallada las características basales de los pacientes del estudio. El $86,93 \%$ de los pacientes con implante definitivo fueron mujeres. La edad media fue de 56,52 años (rango: 22-76 años). El tiempo medio de IF fue de 7,65 años (rango: 9 meses a 18 años). En la tabla IV se muestran los valores basales y durante el seguimiento tras el implante de la NRS, sobre la escala de continencia Wexner-Cleveland y en la capacidad de retraso de la evacuación. No hubo pérdida de ningún paciente durante el seguimiento. El valor medio basal previo a la NRS en la escala Wexner-Cleveland fue de 15,00 $\pm 1,81$. El valor medio en esta escala al año de la NRS fue de 4,87 $\pm 2,54$, obteniéndose una eficacia terapéutica global del $67,5 \%$ con respecto a la disminución en la puntuación sobre la escala Wexner-Cleveland. Por otra parte, antes de la NRS, el 62,50\% de los pacientes tenía una capacidad de retraso de la defecación menor de 1 minuto; sin embargo, al año de la NRS el $75,01 \%$ de los pacientes presentaba una capacidad mayor de 15 minutos.

El electrodo de estimulación definitiva fue colocado en $\mathrm{S} 3$ en 10 pacientes $(38,46 \%)$ y en S4 en $14(61,53 \%)$. La corriente de estimulación empleada fue programada con una frecuencia de $16 \mathrm{~Hz}$ y una amplitud de $210 \mu \mathrm{s}$. En ningún caso, el voltaje fue superior a $1 \mathrm{~V}$ (valor medio: 0,64 V; rango: 0,20-1 V). La estancia hospitalaria postoperatoria fue de 1 día para todos los pacientes. No se presentaron complicaciones graves intraoperatorias ni durante el seguimiento; tampoco se produjeron movilizaciones de los electrodos durante todo el estudio. 
Tabla III. Características basales muestrales

\begin{tabular}{|c|c|c|c|c|c|c|c|c|c|c|c|c|}
\hline $\begin{array}{l}\text { Pac. } \\
\text { núm. }\end{array}$ & $\begin{array}{l}\text { Sexoy } \\
\text { edad } \\
\text { (años) }\end{array}$ & $\begin{array}{l}\text { Antec. } \\
\text { médicos }\end{array}$ & $\begin{array}{c}\text { Antec. } \\
\text { obstetricos }\end{array}$ & $\begin{array}{l}\text { Cirugía pélvica- } \\
\text { perianal }\end{array}$ & $\begin{array}{l}\text { Edad al } \\
\text { inicio de } \\
\text { IF (años) }\end{array}$ & Asocia a: & $\begin{array}{l}\text { Wexner } \\
\text { basal }\end{array}$ & $\begin{array}{l}\text { Hábito } \\
\text { desposi- } \\
\text { cional }\end{array}$ & $\begin{array}{l}\text { Núm. } \\
\text { desposiciones } \\
\text { semanales } \\
\text { medio }\end{array}$ & $\begin{array}{l}\text { Capacidad } \\
\text { retraso } \\
\text { evacuación }\end{array}$ & EEA & $\begin{array}{c}\text { Manometría } \\
\text { (mmHG) } \\
\text { PMBIPMCV }\end{array}$ \\
\hline 1 & Mujer, 70 & & $6 \mathrm{PV}(2 \mathrm{D})$ & HV. Hrr & 55 & Men & 14 & N & 6 & $1-5 \mathrm{~min}$ & $\begin{array}{l}\text { Adelgazam. } \\
\text { ambos esfínteres }\end{array}$ & $44 / 99$ \\
\hline 2 & Mujer, 46 & & $2 \mathrm{PV}$ & ELL. FT & 52 & $\mathrm{FT}$ & 13 & N & 6 & $5-10 \mathrm{~min}$ & $\begin{array}{l}\text { Fibrosis EAE } \\
\text { Defecto ELl. }\end{array}$ & $54 / 94$ \\
\hline 3 & Mujer, 70 & & & Hrr. FT. EP & 40 & Men & 14 & N & 5 & $1-5 \mathrm{~min}$ & Normal & $85 / 109$ \\
\hline 4 & Mujer, 76 & & $3 \mathrm{PV}(1 \mathrm{D})$ & $\begin{array}{c}\text { Pexia pélvica } \\
\text { (cistocele y rectocele) }\end{array}$ & 53 & Men & 13 & $E$ & 4 & $5-10 \mathrm{~min}$ & Normal & $73 / 144$ \\
\hline 5 & Mujer, 41 & & $3 \mathrm{PV}$ & & 54 & $?$ & 14 & A & 7 & $1-5 \mathrm{~min}$ & $\begin{array}{l}\text { Adelgazam. } \\
\text { ambos esfínteres }\end{array}$ & $65 / 98$ \\
\hline 6 & Mujer, 64 & & $3 \mathrm{PV}$ & HV & 58 & Men & 13 & N & 5 & $1-5 \mathrm{~min}$ & Normal & $55 / 72$ \\
\hline 7 & Mujer, 65 & & 1 PV (D) & FT. EP & 49 & $?$ & 16 & N & 6 & $<1 \mathrm{~min}$ & Normal & $45 / 91$ \\
\hline 8 & Mujer, 60 & DM & $2 \mathrm{PV}$ & & 46 & $?$ & 14 & A & 8 & $1-5 \mathrm{~min}$ & Normal & $32 / 76$ \\
\hline 9 & Mujer, 60 & & $2 \mathrm{PV}$ & Hrr & 60 & PP & 13 & N & 5 & $1-5 \mathrm{~min}$ & Normal & $46 / 73$ \\
\hline 10 & Mujer, 61 & DM & & & 56 & $\mathrm{FT}$ & 16 & N & 6 & $<1 \mathrm{~min}$ & Normal & $44 / 98$ \\
\hline 11 & Mujer, 75 & & $7 \mathrm{PV}$ & HV. Pexia pélvica & 55 & Men & 18 & N & 6 & $<1 \mathrm{~min}$ & Normal & $53 / 59$ \\
\hline 12 & Mujer, 71 & & $1 \mathrm{PV}$ & & 65 & Men & 13 & N & 4 & $1-5 \mathrm{~min}$ & Normal & $54 / 71$ \\
\hline 13 & Mujer, 38 & & $3 \mathrm{PV}$ & Desgarro perineal. EP & 33 & PP & 15 & N & 5 & $<1 \mathrm{~min}$ & Fibrosis EAE & $45 / 120$ \\
\hline 14 & Mujer, 37 & & $1 \mathrm{PV}(\mathrm{D})$ & FT & 31 & Men & 14 & N & 6 & $<1 \mathrm{~min}$ & Normal & $30 / 60$ \\
\hline 15 & Hombre, 22 & $\begin{array}{l}\text { Atresia anal } \\
\text { congénita }\end{array}$ & & $\begin{array}{c}\text { Descenso rectal } \\
\text { sin sección de EAE. } \\
\text { Cierre colostomía } \\
\text { terminal }\end{array}$ & 19 & $\begin{array}{l}\text { Reconstr. } \\
\text { atresia anal }\end{array}$ & 20 & N & 9 & $<1 \mathrm{~min}$ & Normal & $52 / 123$ \\
\hline 16 & Mujer, 56 & & $3 \mathrm{PV}$ & & 51 & $\mathrm{FT}$ & 17 & N & 6 & $<1 \mathrm{~min}$ & Normal & $60 / 0$ \\
\hline 17 & Mujer, 29 & $\begin{array}{l}\text { Polineuropat. } \\
\text { periférica idiopática }\end{array}$ & $1 \mathrm{PV}(\mathrm{D})$ & EP & 28 & PP & 16 & A & 6 & $<1 \mathrm{~min}$ & Fibrosis EAE & $30 / 60$ \\
\hline 18 & Hombre, 44 & DM & & & 41 & Viaje pais tropical & 14 & $E$ & 4 & $1-5 \mathrm{~min}$ & Normal & $32 / 40$ \\
\hline 19 & Mujer, 75 & & $3 P V(1 D)$ & HV & 59 & Men & 13 & $E$ & 4 & $<1 \mathrm{~min}$ & $\begin{array}{l}\text { Adelgazam. } \\
\text { ambos esfínteres }\end{array}$ & $46 / 74$ \\
\hline 20 & Mujer, 70 & & $2 \mathrm{PV}$ & $\mathrm{Hrr}$ & 58 & Men & 13 & N & 5 & $1-5 \mathrm{~min}$ & Normal & $75 / 91$ \\
\hline 21 & Mujer, 66 & & $4 \mathrm{PV}$ & ELI & 55 & $?$ & 13 & N & 6 & $<1 \mathrm{~min}$ & Defecto EAl & $54 / 94$ \\
\hline 22 & Hombre, 55 & & & $\mathrm{FT}$ & 54 & FT & 16 & N & 6 & $<1 \mathrm{~min}$ & Normal & $55 / 71$ \\
\hline 23 & Mujer, 33 & & $1 \mathrm{PV}(\mathrm{D})$ & $\begin{array}{l}\text { Desgarro } \\
\text { perineal. EP }\end{array}$ & 33 & PP & 17 & N & 6 & $<1 \mathrm{~min}$ & Normal & $30 / 62$ \\
\hline 24 & Mujer, 64 & & $1 \mathrm{PV}$ & HV & 57 & Men & 14 & N & 7 & $<1 \mathrm{~min}$ & Normal & $43 / 89$ \\
\hline 25 & Mujer, 71 & DM & $3 \mathrm{PV}$ & & 59 & Men & 13 & N & 6 & $1-5 \mathrm{~min}$ & Normal & $44 / 97$ \\
\hline 26 & Mujer, 76 & & 1 PV & HV & 49 & $?$ & 14 & N & 6 & $<1 \mathrm{~min}$ & Normal & $60 / 0$ \\
\hline Media & 56,5 & DM: 15,38\% & $\begin{array}{c}P V: 53 \\
D: 1509 \%\end{array}$ & $\begin{array}{l}\text { ELl: 7,69\% } \\
\text { EP: 7,69\% }\end{array}$ & & $\begin{array}{l}\text { FT: } 15,38 \% \\
\text { Men: } 42,3 \%\end{array}$ & & $\begin{array}{l}N: 80,77 \% \\
E: 11,54 \%\end{array}$ & & $\begin{array}{l}<1 \min : 53,8 \% \\
1-5 \min : 38,5 \%\end{array}$ & $\begin{array}{l}N: 76,93 \% \\
A: 23,07 \%\end{array}$ & $50,23 / 80,58$ \\
\hline & & & & $\begin{array}{l}\text { FT: } 19,23 \% \\
\text { Hrr: } 15,38 \% \\
\text { HV: } 19,23 \% \\
\text { Otros: } 11,54 \%\end{array}$ & 48,84 & $\begin{array}{c}\text { PP: } 15,38 \% \\
?: 19,23 \% \\
\text { Otros: } 7,71 \%\end{array}$ & 14,6 & A: $7,69 \%$ & 5,8 & $\begin{array}{l}5-10 \min : 7,7 \% \\
10-15 \min : 0 \% \\
>15 \min : 0 \%\end{array}$ & & \\
\hline
\end{tabular}

A: alterno; D:distócico; DM: diabetes mellitus; E: estreñimiento; EAE: esfínter anal externo; EAl: esfínter anal interno; EEA:ecografía endoanal; ELI: esfinterotomía lateral interna; EP: esfinteroplastia; FT: cirugía fístula anal compleja; Hrr: hemorroidectomía; Men: menopausia; N: normal; PP: postparto; ?: no recuerda.

Fue necesario recolocar la batería de electroestimulación subcutánea en una paciente a los 9 meses de su implante, debido a una notable pérdida de peso que condicionaba que la batería interna quedase excesivamente superficial y molesta para la paciente.

\section{DISCUSIÓN}

El aumento de la demanda asistencial de la IF debido tanto al envejecimiento en las pirámides poblacionales de los países desarrollados, como al mayor acceso a la infor- mación médica, ha condicionado un resurgimiento en el interés médico en esta patología, en el que los recursos terapéuticos históricamente han sido limitados y poco efectivos $(1-4,6)$. Tres son los mecanismos implicados en la continencia fecal: un sistema valvular esfintérico competente, un reservorio rectal distensible y una adecuada inervación de dichas estructuras, interconectada a su vez con el sistema nervioso central para hacer consciente y voluntario el acto defecatorio $(17,19)$. En este sentido, ante la existencia de un defecto anatómico esfintérico, la esfinteroplastia clásica constituye la opción quirúrgica indicada $(4,5)$; si a pesar de esta persiste dicho defecto, se 
Tabla IV. Resultados

\begin{tabular}{|c|c|c|c|c|c|c|c|c|c|c|}
\hline $\begin{array}{l}\text { Núm } \\
\text { pac. }\end{array}$ & $\begin{array}{c}\text { Wexner } \\
\text { basal }\end{array}$ & $\begin{array}{l}\text { Wexner } \\
\text { al mes } \\
\text { de NRS }\end{array}$ & $\begin{array}{l}\text { Wexner a } \\
\text { los } 6 \text { meses } \\
\text { de NRS }\end{array}$ & $\begin{array}{l}\text { Wexner al } \\
\text { año de NRS }\end{array}$ & $\begin{array}{c}\text { Estudio esta- } \\
\text { distico (rangos y } \\
\text { signos de Wilcoxon } \\
\text { y Mann-Whitney) } \\
\text { Valor de " } p \text { " }\end{array}$ & $\begin{array}{l}\text { Capacidad } \\
\text { retraso } \\
\text { evacuación } \\
\text { basal }\end{array}$ & $\begin{array}{l}\text { Capacidad } \\
\text { retraso } \\
\text { evacuación } \\
\text { al mes } \\
\text { de NRS }\end{array}$ & $\begin{array}{c}\text { Capacidad } \\
\text { retraso } \\
\text { al mes } \\
\text { de NRS }\end{array}$ & $\begin{array}{l}\text { Capacidad } \\
\text { retraso } \\
\text { a los } 6 \\
\text { meses de } \\
\text { NRS }\end{array}$ & $\begin{array}{c}\text { Estudio } \\
\text { estadistico } \\
\text { (Chi-cuadrado } \\
\text { de Pearson) } \\
\text { Valor de " } x^{2 "}\end{array}$ \\
\hline 1 & 14 & 3 & 2 & 2 & & $1-5 \mathrm{~min}$ & $>15 \mathrm{~min}$ & $>15 \mathrm{~min}$ & $>15 \mathrm{~min}$ & \\
\hline 2 & 13 & 3 & 3 & 2 & & 5-10 min & $>15 \mathrm{~min}$ & $>15 \mathrm{~min}$ & $>15 \mathrm{~min}$ & \\
\hline 3 & 14 & 5 & 6 & 6 & & $1-5 \min$ & $>15 \mathrm{~min}$ & $>15 \mathrm{~min}$ & $>15 \mathrm{~min}$ & \\
\hline 4 & 13 & 6 & 7 & 6 & & $5-10 \mathrm{~min}$ & $>15 \mathrm{~min}$ & $>15 \mathrm{~min}$ & $>15 \mathrm{~min}$ & \\
\hline 5 & 14 & 3 & 3 & 9 & & $1-5 \mathrm{~min}$ & $5-10 \mathrm{~min}$ & $5-10 \mathrm{~min}$ & $10-15 \mathrm{~min}$ & \\
\hline 6 & 13 & 6 & 5 & 4 & & $1-5 \mathrm{~min}$ & $10-15 \mathrm{~min}$ & $10-15 \mathrm{~min}$ & $10-15 \mathrm{~min}$ & \\
\hline 7 & 16 & 3 & 2 & 9 & & $<1 \min$ & 5-10 min & 5-10 min & 5-10 min & \\
\hline 8 & 14 & 2 & 2 & 2 & & $1-5 \mathrm{~min}$ & $>15 \mathrm{~min}$ & $>15 \mathrm{~min}$ & $>15 \mathrm{~min}$ & \\
\hline 9 & 13 & 6 & 6 & 5 & & $1-5 \min$ & $>15 \mathrm{~min}$ & $>15 \mathrm{~min}$ & $>15 \mathrm{~min}$ & \\
\hline 10 & 16 & 4 & 4 & 4 & & $<1 \min$ & $>15 \min$ & $>15 \mathrm{~min}$ & $>15 \mathrm{~min}$ & \\
\hline 11 & 18 & 5 & 3 & 4 & & $<1 \min$ & $>15 \mathrm{~min}$ & $>15 \mathrm{~min}$ & $>15 \mathrm{~min}$ & \\
\hline 13 & 15 & 6 & 6 & 6 & & $<1 \min$ & $5-10 \mathrm{~min}$ & $5-10 \mathrm{~min}$ & $5-10 \mathrm{~min}$ & \\
\hline 14 & 14 & 3 & 4 & 5 & & $<1 \min$ & $>15 \mathrm{~min}$ & $>15 \mathrm{~min}$ & $>15 \mathrm{~min}$ & \\
\hline 15 & 20 & 9 & 11 & 10 & & $<1 \min$ & $5-10 \mathrm{~min}$ & $5-10 \mathrm{~min}$ & $5-10 \mathrm{~min}$ & \\
\hline 16 & 17 & 4 & 4 & 5 & & $<1 \min$ & $5-10 \mathrm{~min}$ & $5-10 \mathrm{~min}$ & $5-10 \mathrm{~min}$ & \\
\hline 17 & 17 & 5 & 5 & 4 & & $<1 \min$ & $10-15 \mathrm{~min}$ & $>15 \mathrm{~min}$ & $>15 \mathrm{~min}$ & \\
\hline 19 & 19 & 5 & 6 & 6 & & $<1 \min$ & $>15 \mathrm{~min}$ & $>15 \mathrm{~min}$ & $>15 \mathrm{~min}$ & \\
\hline 20 & 13 & 5 & 4 & 4 & & $<1 \min$ & $>15 \mathrm{~min}$ & $>15 \mathrm{~min}$ & $>15 \mathrm{~min}$ & \\
\hline 21 & 13 & 4 & 4 & 4 & & $<1 \min$ & $>15 \min$ & $>15 \min$ & $>15 \mathrm{~min}$ & \\
\hline 22 & 16 & 4 & 5 & 4 & & $<1 \min$ & $>15 \mathrm{~min}$ & $>15 \mathrm{~min}$ & $>15 \mathrm{~min}$ & \\
\hline 23 & 17 & 4 & 3 & 2 & & $<1 \min$ & $>15 \mathrm{~min}$ & $>15 \mathrm{~min}$ & $>15 \mathrm{~min}$ & \\
\hline 24 & 14 & 6 & 7 & 6 & & $<1 \min$ & $>15 \min$ & $>15 \min$ & $>15 \mathrm{~min}$ & \\
\hline 25 & 13 & 6 & 5 & 6 & & $1-5 \mathrm{~min}$ & $>15 \mathrm{~min}$ & $>15 \mathrm{~min}$ & $>15 \mathrm{~min}$ & \\
\hline 26 & 14 & 4 & 2 & 2 & & $<1 \min$ & $>15 \mathrm{~min}$ & $>15 \min$ & $>15 \mathrm{~min}$ & \\
\hline \multirow[t]{5}{*}{ Media } & 15 & 4,62 & 4,54 & 4,87 & 0,0031 & $<1 \min : 62,50 \%$ & $<1 \min : 0 \%$ & $<1 \mathrm{~min}: 0 \%$ & $<1 \min : 0 \%$ & 0,0018 \\
\hline & & & & & & $1-5 \min : 29,17 \%$ & $1-5 \min : 0 \%$ & $1-5 \min : 0 \%$ & $1-5 \min : 0 \%$ & \\
\hline & & & & & & $5-10 \min : 8,33 \%$ & $5-10$ min: $20.84 \%$ & $5-10 \min : 20.83 \%$ & $5-10 \min : 16,66 \%$ & \\
\hline & & & & & & 10-15min: $0 \%$ & 10-15min: 8,33\% & 10-15min: 4,16\% & 10-15min: $8,33 \%$ & \\
\hline & & & & & & $>15$ min: $0 \%$ & $>15 \min : 70,83 \%$ & $>15$ min: $75,01 \%$ & $>15$ min: $75,01 \%$ & \\
\hline
\end{tabular}

recurrirá a técnicas quirúrgicas más complejas y no exentas de complicaciones, como la graciloplastia dinámica y el esfínter artificial $(6,17,20-23)$. La NRS surge como resultado del desarrollo de la neurofisiología defecatoria, como una opción terapéutica para los casos con IF sin defecto anatómico objetivable, en los que históricamente ha existido un vacío terapéutico $(6,10,17)$. La NRS, mediante la estimulación eléctrica de las fibras sensoriales aferentes sacras, consigue una mejora en el equilibrio de interacción entre las distintas estructuras implicadas en el mecanismo defecatorio, actuando sobre centros reflejos neurológicos (no sólo medulares, sino también pontinos), con repercusiones a nivel de la contractilidad, distensibilidad y sensibilidad rectal $(24,25)$. Así, a través de la estimulación de fibras aferentes sacras, se produce una neuromodulación sobre los centros reflejos defecatorios, dando como resultado una mejora sobre la continencia anal $(6,10,17)$. Para que la NRS sea efectiva, se requiere de una integridad en el sistema de inervación de las estructuras implicadas en el mecanismo defecatorio y de la ausencia de defectos anatómicos esfinterianos $(2,6,9,10)$. Por otra parte, el debilitamiento de las estructuras impli- cadas en la defecación debido al paso del tiempo y a injurias locales (como los partos vaginales), provoca que el control regulatorio neurológico de la defecación se torne insuficiente, apareciendo la IF. En este sentido, la NRS reactivaría y modularía la interacción de los centros neurológicos defecatorios, resultando una mejora en la continencia. La NRS es una terapia de reciente desarrollo y la bibliografía acerca de esta no es extensa (9). Las series publicadas, todas ellas con escaso número de pacientes, muestran resultados similares en cuando a índices de eficacia en torno al 70\% (13-15,26-28). En nuestra serie, la NRS ha supuesto una reducción media del valor de continencia en la escala de continencia de Cleveland-Wexner del $67,53 \%$, resultado equiparable a los objetivados por otros grupos. La elección de la raíz sacra en la que se implanta el electrodo es variable según las series. A pesar de que la mayoría de estudios sobre NRS para el tratamiento de la incontinencia urinaria recomienda la estimulación de S3 (13-15), Matzel y cols. (29) ponen de manifiesto la importancia de $\mathrm{S} 4$ con respecto a la inervación de la sensibilidad ano-rectal. Por ello, la tendencia actual es la de estimular S4 en los casos de IF y de S3 en los que además 
coexista incontinencia urinaria. No obstante, la decisión última en cuanto a la raíz sacra a estimular debe tomarse en quirófano durante la fase de estimulación aguda, en la cual se seleccionará finalmente la raíz en la que se obtenga una mejor respuesta sensitivo-motora con un mínimo voltaje excitatorio. Recomendamos que el voltaje de la corriente eléctrica empleada para la NRS, sea aquel capaz de producir respuesta sensitiva sin llegar al umbral del dolor o la respuesta motora (12). Durante el seguimiento de los pacientes de nuestra serie, algunos pacientes han requerido un mínimo aumento de dicho voltaje sin conocerse actualmente las implicaciones que esto pueda tener sobre la efectividad futura de la NRS. Una cuestión no aclarada hasta ahora es la discordancia en cuanto a la mejoría en los resultados manométricos tras la NRS en casos con buena respuesta terapéutica. Muy posiblemente, la gran variabilidad individual de cada paciente durante la realización de la manometría sea un factor determinante en este sentido. Así, el método más importante para la evaluación de la efectividad de la NRS como terapia para la IF, es el seguimiento clínico, siendo de vital importancia la confección de diarios de continencia sencillos y a la vez detallados, para objetivar de manera precisa el grado de IF, así como el hábito y la consistencia defecatoria. No obstante, no cabe duda de que nos encontramos ante una terapia eficaz y con un prometedor futuro, que las series extensas con seguimiento a largo plazo acabarán por situarla en un lugar predominante dentro del arsenal quirúrgico para el tratamiento de la IF sin defectos anatómicos objetivables, con escasa complejidad y morbilidad, siendo posible su realización bajo anestesia local y en un régimen de cirugía ambulatorio (17). Finalmente, mostra-

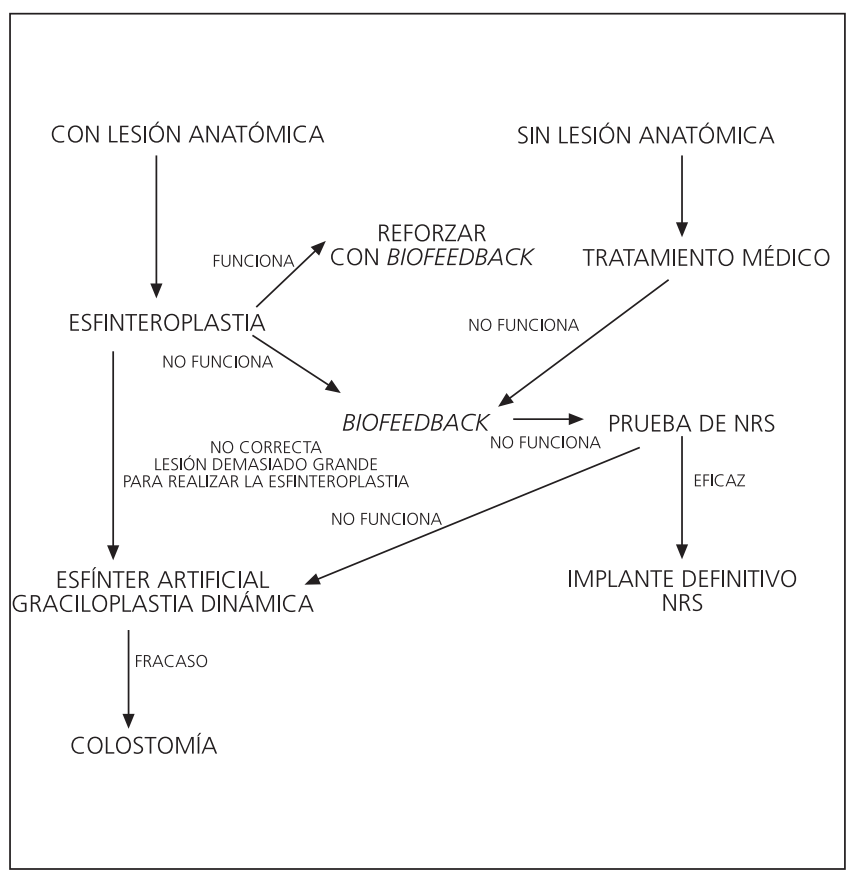

Fig. 1. Algoritmo terapéutico de la incontinencia fecal. mos nuestro algoritmo terapéutico para el tratamiento de la IF, el cual se rige por la existencia o no de defecto anatómico esfinteriano (Fig. 1). En los casos en los que la NRS no sea resolutiva, y a pesar de que no exista defecto anatómico objetivable, se recurrirá a técnicas quirúrgicas anatómicas de segundo orden, tales como la graciloplastia dinámica o la colocación de un esfínter artificial. No obstante, existe una minoría de pacientes que continúa incontinente a pesar de todas las posibles opciones terapéuticas y con severas alteraciones en su calidad de vida. En este sentido, consideramos que la creación de una colostomía ha de ser una opción a proponer al paciente.

\section{BIBLIOGRAFÍA}

1. Whitehead WE, Wald A, Diamant NE, Enck P, Pemberton JH, Rao SS. Functional disorders of the anus and rectum. Gut 1999; 45 (Supl. 2): II55-II59.

2. Ballester A, Minués M, Herreros B. Prevalencia de la incontinencia anal y urinaria silentes en mujeres de la ciudad de Teruel. Rev Esp Enferm Dig 2005; 97 (2): 78-86.

3. Nelson R, Norton N, Cautley E, Furner S. Community-based prevalence of anal incontinence. JAMA 1995; 274: 559-61.

4. Aspiroz F. Guía práctica sobre incontinencia anal. Rev Esp Enferm Dig 2003; 95: 722-6.

5. Bachoo P, Brazzelli M, Grant A. Surgery for faecal incontinence in adults. Cochrane Database Syst Rev 2000; (2): CD001757.

6. Alós R, Solana A, Ruiz MD, Moro D, García Armengol J, Roig JV. Técnicas novedosas en el tratamiento de la incontinencia anal. Cir Esp 2005; 78 (Supl. 3): 41-9.

7. Tanagho EA, Schmidt RA, Orvis BR. Neural stimulation for control of voiding dysfunction: A preliminary report in 22 patients with serious neuropathic voiding disorders. J Urol 1989; 142: 340.

8. Schmidt RA, Senn E, Tanagho EA. Functional evaluation of sacral nerve root integrity. Report of a technique. Urology 1990; 35: 388-92.

9. Bosch JL, Groen J. Sacral (S3) segmental nerve stimulation as a treatment for urge incontinence in patients with detrusor instability: Results of chronic electrical stimulation using an implantable neural prosthesis. J Urol 1995; 154: 504-7.

10. Matzel KE, Stadelmaier U, Hohenfeller M, Gall FP. Electrical stimulation of spinal nerves for treatment of faecal incontinence. Lancet 1995; 346: 1124-7.

11. Muñoz-Duyos A, Montero J, Navarro A, Del Rio C, García-Domingo MI, Marco C. Incontinencia fecal: Neurofisiología y neuromodulación. Cir Esp 2004; 76 (2): 65-70.

12. Malouf AJ, Vaizey CJ, Nicholls RJ, Kamm MA. Permanent sacral nerve stimulation for faecal incontinence. Ann Surg 2000; 232: 143-8.

13. Ganio E, Realis A, Clerico G, Trompetto M. Sacral nerve stimulation for treatment of faecal incontinence. Dis Colon Rectum 2001; 44 : 619-31.

14. Leroi AM, Michot F, Grise P, Denis P. Effect of sacral nerve stimulation in patients with fecal and urinary incontinence. Dis Colon Rectum 2001; 44: 779-89.

15. Ganio E, Ratto C, Masin A, et al (GINS experience). Neuromodulation for fecal incontinence: outcome in 16 patients with definitive implant. Dis Colon Rectum 2001; 44: 965-70.

16. Kenefick NJ, Vaizey CJ, Cohen RCG, Nicholls RJ, Kamm MA. Medium-term results of permanent sacral nerve stimulation for faecal incontinence. Br J Surg 2002; 89: 896-901.

17. Linares Quevedo AI, Jiménez Cidre MA, Fernández Fernández E, et al. Neuromodulación de raíces sacras posteriores en el tratamiento de la disfunción miccional crónica. Actas Urol Esp 2002; 26 : 250-60.

18. Muñoz-Duyos A, Navarro A, Rius J, Martí-Gallostra M, Marco C. Estimulación de raíces sacras como tratamiento de la incontinencia fecal. Resultados preliminares. Cir Esp 2004; 76 (3): 169-76. 
19. Rockwood TH, Church JM, Fleschman JW, et al. Fecal incontinence Quality of Life Scale. Dis Colon Rectum 2000; 43: 9-17.

20. Orrom WJ, Miller R, Cornes H, Duthie G, Mortenson NJ, Bartolo DCC. Comparison of anterior sphinteroplasty and post anal repair in the treatment of idiophatic fecal incontinence. Dis Colon Rectum 1991; 34: 305-10.

21. Baeten CG, Consten J, Spans F, et al. Dynamic graciloplasty for treatment of faecal incontinence. Lancet 1991; 338: 1163-5.

22. Christiansen J, Lorentzen M. Implantation of artificial sphincter for anal incontinence: Report of five cases. Dis Colon Rectum 1989; 32: 432-6.

23. Lehur PA, Michot F, Denis P. Results of artificial sphincter in severe anal incontinence: Report of 14 consecutive implantations. Dis Colon Rectum 1996; 39: 1352-5.

24. Wexner SD, Baeten C, Bauley R, et al. Long-term efficacy of dynamic graciloplasty for fecal incontinence. Dis Colon Rectum 2002; 45 : 809-18.
25. Vaizey CJ, Kamm MA, Turner IC, Nicholls RJ, Woloszko J. Effects of short-term sacral nerve stimulation on anal and rectal function in patients with anal incontinence. Gut 1999; 44: 407-12.

26. Kenefick NJ, Nicholls RJ, Cohen RG, Kamm MA. Permanent sacral nerve stimulation for treatment of idiopathic constipation. Br J Surg 2002; 89: 882-8.

27. Rosen HR, Urbarz C, Holzer B, Novi G, Schiessel R. Sacral nerve stimulation as a treatment for fecal incontinence. Gastroenterol 2001; 121: 536-41.

28. Kenefick NJ, Vaizey CJ, Cohen RCG, Nicholls RJ, Kamm MA. Medium-term results of permanent sacral nerve stimulation for faecal incontinence. Br J Surg 2002; 89: 896-9.

29. Matzel KE, Schmidt RA, Tanagho EA. Neuroanatomy of the striated muscular anal continence mechanism. Implications for the use of neurostimulation. Dis Colon Rectum 1990; 33: 66673. 